\title{
Disposición de los latinoamericanos hacia la protesta. Un análisis exploratorio a partir de Latinobarómetro $2015^{1}$
}

\author{
Willingness of Latin Americans to protest. \\ An exploratory analysis using Latinobarómetro 2015 \\ RODRIGO CUEVAS OSSANDÓN ${ }^{2}$ \\ Universidad de Chile \\ CRISTOBAL VILLALOBOS DINTRANS ${ }^{3}$ \\ Centro de Estudios de Políticas y Prácticas en Educación (CEPPE-UC)
}

RECEPCIÓN：02/10/2017 • ACEPTACIÓN: 29/10/2017

RESUMEN: Considerando el contexto de crecimiento económico y relativa estabilidad democrática que ha experimentado América Latina y el Caribe durante la última década, resulta paradójico el aumento de la cantidad y magnitud de protestas que se ha experimentado en el continente. Como una forma de entregar información que permita entender esta paradoja, el artículo busca analizar la disposición a protestar por parte de los latinoamericanos, usando los datos de la encuesta Latinobarómetro

1. Se agradecen las valiosas observaciones entregadas por un evaluador anónimo, las que permitieron enriquecer la discusión teórica, precisar el diseño metodológico y con ello la interpretación de los resultados. Esperamos haber podido incorporar de la mejor manera posible sus valiosas contribuciones. Adicionalmente, Cristóbal Villalobos agradece el patrocinio brindado por el Centro de Estudios del Conflicto y la Cohesión Social (COES), Proyecto CONICYT/ FONDAP/15130009.

2. Magíster en Estudios Internacionales, Universidad de Chile. Becario de Doctorado Nacional, CONICYT, en Programa de Ciencias Sociales de la Universidad de Chile. email: rodrigocuevas@ uchile.cl

3. Sociólogo y Trabajador Social de la Pontificia Universidad Católica de Chile. Magíster en Economía Aplicada a las Políticas Públicas de a Universidad Alberto Hurtado-Georgetown University. Doctor @ en Ciencias Sociales de la Universidad de Chile. email: clvillal@uc.cl 
2015. A partir de la revisión de literatura, se consideran cuatro grupos de factores: i) uso de tecnología; ii) valoración de la democracia; iii) ejercicio de la ciudadanía y; iv) variables sociodemográficas. Los resultados muestran que muchas de las variables tradicionales utilizadas para explicar la acción de la protesta no predicen de manera efectiva la disposición de esta. No obstante, la participación en procesos democráticos y la valoración de la democracia aparecen como variables relevantes y significativas para explicar esta disposición. Finalmente, se observa una alta variabilidad entre países respecto del peso de estos factores, siendo el modelo ajustado para explicar la disposición a protestar en países donde han existido altos niveles de acción colectiva, como Chile y Brasil.

PALABRAS CLAVE: Acción Colectiva - Opinión Pública - Democracia - América Latina - Protesta.

ABSTRACT: Considering the context of economic growth and relative democratic stability that Latin American and Caribbean countries have enjoyed during the last decade, the increase in the quantity and magnitude of protests that has been experienced in the continent appears paradoxical. As a way of obtaining information to help understand this paradox, the present article seeks to investigate the factors that would explain the willingness of Latin Americans to protest, using data from the Latinobarometer 2015 Survey. Four groups of factors are considered: i) use of technology; ii) appreciation of democracy; iii) practice of citizenship and; iv) socio-demographic variables. The results show that many of the traditional variables used to explain protest actions do not effectively predict the willingness to protest. Nevertheless, participation in democratic processes and the evaluation of democracy are both relevant and significant in explaining this willingness. Finally, there is great variability between countries; the model has therefore been adjusted to explain the willingness to protest in countries where there have been high levels of collective action, such as Chile and Brazil.

KEYWORDS: Collective Action - Public Opinion - Democracy - Latin America - Protest. 


\section{Introducción y problematización}

En la última década, los países de América Latina y el Caribe (LAC) presentaron un aumento en sus protestas, tanto en la frecuencia de su ocurrencia como en el alcance que estas adquirieron, configurando lo que Roberts (2017) ha denominado como la "tercera ola" de protestas en la región. Así, entre 2006 y 2013, la región ocupó el segundo lugar en la mayor cantidad de protestas, siendo solo superado por el caso de los países de altos ingresos (Ortiz, Burke, Berrada y Cortés, 2013).

A diferencia de otros momentos, este ciclo de protestas se desarrolla en condiciones bastante particulares. En primer lugar, la región no se vio en demasía afectada por eventos de constricción económica, como la recesión mundial del año 2008, que llevó a la adopción de medidas de austeridad en parte importante de los países del mundo (Krugman, 2012; Stiglitz, 2012; Ortiz, Burke Berrada y Cortés, 2013). Por el contrario, esta ola de protestas se da, en el caso latinoamericano, en un contexto de mejoras en el bienestar económico de sus ciudadanos, pese a que estos efectos virtuosos se revirtieron parcialmente a partir de 2015 (CEPAL, 2017). Así, y tomando el año 2000 como referencia, el Producto Interno Bruto (PIB) per cápita de los 20 países principales países de la región ${ }^{4}$ era un $13 \%$ superior en 2006, un $25 \%$ en 2010, siendo finalmente en 2013 un 37\% mayor (Cuadro 1). Este avance también se registró en materias históricamente problemáticas para América Latina, como la distribución del ingreso, la que si bien sigue siendo una de las más desiguales a nivel mundial, ha disminuido en los últimos años (CEPAL, 2017, p. 14).

En segundo término, este aumento de la conflictividad y las protestas se ha desarrollado en un contexto de relativa consolidación de sus regímenes democráticos. Así, en este período, no se presentarían en demasía problemas con la democracia, en el sentido de un peligro de golpe militar, fenómeno bastante recurrente a lo largo de todo el siglo XX en la región ${ }^{5}$. Al contrario, durante el período se ha puesto en discusión la capacidad de los países de la región de dar cuenta de las demandas ciudadanas y de profundizar la calidad de sus democracias (PNUD, 2004; PNUD, 2007; Diamond \& Morlino, 2005; Altman y Pérez-Liñán, 2002), relevando la crisis

4. Se compilaron datos para los siguientes países: Argentina, Bolivia, Brasil, Chile, Colombia, Costa Rica, Cuba, República Dominicana, Ecuador, El Salvador, Guatemala, Honduras, Jamaica, México, Nicaragua, Panamá, Paraguay, Perú, Uruguay y Venezuela.

5. Sin embargo, durante los últimos años se han producido procesos de ruptura democrática en varios países de la región, como Honduras, Brasil o Paraguay. Adicionalmente, otros países, como Venezuela, han experimentado importantes crisis a su sistema democrático. 
de funcionamiento entre instituciones existente en varios países de la región, lo que en algunos casos han derivado en el término anticipado de presidencias, pero sin una retroceso hacia un autoritarismo del estilo que se observó en el siglo pasado (O`Donell, 1994; Pérez-Liñán, 2009; Martínez, 2015).

Este contexto positivo en términos económicos e institucionales lleva a preguntarnos qué explicaría el aumento de las protestas en la región y cuáles serían los principales factores que podrían explicar la disposición a protestar, lo que constituye el objetivo principal de este artículo.

Las explicaciones que se han dado hasta la fecha han sido diversas. En algunos casos, se ha apuntado a la capacidad de los gobiernos en proveer un buen funcionamiento de los servicios públicos (Ortiz, Burke, Berrada y Cortés, 2013) como el factor clave de este ciclo de protestas, lo que representaría un cambio respecto a mediados de la década de los 90 y comienzos del 2000, donde las principales protestas en la región eran respecto al avance de las reformas neoliberales (Almeida, 2007, pp. 134-135). En otros casos, se ha indicado que la mayor diferencia estaría en la novedad del tipo de actor que canalizaría estas demandas, que sería en este caso las clases medias beneficiadas por esta bonanza económica (Bertelsmann Stiftung, 2017). Finalmente, también se ha indicado que este ciclo de protestas podría estar producido por un fenómeno de repolitización de la ciudadanía, que, en contextos de liberalización económica y política, generaría un aumento de las protestas(Bellinger y Arce, 2011, pp. 699-702).

\section{Cuadro 1.}

Número de protestas, PIB per cápita y democracia en América Latina

\begin{tabular}{llllll}
\hline & \multirow{2}{*}{$\begin{array}{l}\text { Eventos de } \\
\text { protestas } \\
\text { masivas }\end{array}$} & PIB per cápita & $\begin{array}{l}\text { Crecimiento } \\
\text { Anual }\end{array}$ & $\begin{array}{l}\text { Proporción } \\
\text { respecto a 2000 }\end{array}$ & $\begin{array}{l}\text { Índice de } \\
\text { Democracia }\end{array}$ \\
\cline { 3 - 5 } Año & 14 & 5936 & 5,0 & 1,13 & 7.3 \\
\hline 2006 & 14 & 6258 & 5,4 & 1,20 & 7.3 \\
2007 & 12 & 5459 & $-12,8$ & 1,04 & 7.3 \\
2008 & 15 & 6309 & 15,6 & 1,21 & 6.9 \\
2009 & 12 & 6548 & 3,8 & 1,25 & 6.9 \\
2010 & 21 & 6801 & 3,9 & 1,30 & 6.9 \\
2011 & 22 & 6979 & 2,6 & 1,33 & 6.9 \\
2012 & 25 & 7152 & 2,5 & 1,37 & 7.3 \\
\hline
\end{tabular}

* El PIB per cápita promedio en 2000 era de 5.234 USD de 2010.

Fuente: World Development Indicators, Banco Mundial; (Marshall, 2013); (Ortiz, Burke, Berrada y Cortés, 2013). 
En síntesis, los antecedentes expuestos muestran que la protesta en la región se ha desarrollado con cambios en sus magnitudes, actores, demandas y formas de desarrollo en la última década. En general, la literatura académica ha buscado entender este proceso como parte de cambios en los procesos de desarrollo de la región, o bien como parte de transformaciones estructurales de América Latina. En el primer caso, el desarrollo de las protestas aparece como una consecuencia de tensiones producidas por procesos de cambio económico o político, que pueden estar influidos por la modernización o liberalización económicas (King y Pearce, 2010; Almeida, 2007; Von Büllow, 2009; Eisenstadt, 1968) o por la resistencia frente a autoritarismos ${ }^{6}$ (Roberts y Garton Ash, 2009). En el segundo caso, la protesta se entiende desde la óptica de los movimientos sociales, enfatizando el carácter estructural del cambio (Garretón, 2002), la coyuntura que hace posible la protesta (Donoso, 2013) o considerando otro factor que influye en esta agregación colectiva, como por ejemplo, su alcance transnacional (Glasius y Pleyers, 2013; Von Büllow, 2009).

A diferencia de estas visiones, es posible reconocer una tercera aproximación, donde el foco está puesto en la protesta como un objeto de estudio en sí mismo. En este caso, la unidad de análisis es el evento de protesta, el que es reconocido como un fenómeno social diferenciado y particular (Olzak, 1989; Koopmans y Rucht, 2002).En algunos casos, el foco del análisis está puesto en el registro de los eventos (Doherty, Plows y Wall, 2007), en los determinantes que lo explicarían (Almeida, 2007), en el rol de los partidos políticos en estos procesos (Arce, 2010)o en una mayor probabilidad de ocurrencia de un determinado modo en la esta se expresa por sobre otro (Medel y Somma, 2016).

Dentro de esta tercera línea, que es desde la cual desarrollamos el presente artículo, nos centraremos en el entendimiento de los factores individuales que pueden estar influyendo en la disposición a protestar. Aunque existe una nutrida investigación que ha indagado en este grupo de factores, por ejemplo, analizando los aspectos psicosociales que determinarían la participación en las protestas (Klandermans, 2007), el rol de las redes sociales (Diani y Mische, 2015), el rol de las emociones (Flam, 2015) o los costos individuales involucrados en las decisiones de participar en acciones colectivas (Finkel y Muller, 1998), en nuestro caso

6. Utilizamos este término a partir de los casos analizados en el libro editado por Adam Roberts y Timothy Garton Ash, en los que si bien la mayoría de los casos analizados corresponden a dictaduras, también se presentan experiencias de presidentes electos que han devenido en autoritarismos (Roberts y Garton Ash, 2009). 
buscaremos entender que factores podrían estar influyendo en el paradojal contexto latinoamericano anteriormente descrito.

Adicionalmente, y a diferencia de otras investigaciones, nos centraremos en entender la disposición a las protestas más que la participación misma en estas acciones colectivas. Esto es relevante, pues las protestas no solo expresan procesos estructurales de transformación societal, sino que también son catalizadores de acción motivados por la posición y disposición que los sujetos tienen en la estructura social (Wulff, Berstein y Taylor, 2015), por la capacidad que tienen estos procesos de transformar las relaciones sociales, afectivas y cognitivas de los sujetos (Della Porta, 2008), por la capacidad de catalizar emociones como la ira, el medio o la desesperanza (Castells, 2012) o por la posibilidad de generar transformaciones personales en los actores, detonando procesos de cambio a la vez, personales (micro) y colectivos (macro)(Goodwin, Jasper y Polleta, 2007).

De esta manera, optamos por estimar lo que sería una disposición a protestar porque si bien podemos conocer qué lleva a una persona a protestar, como por ejemplo por medio del efecto de otros participantes sobre este (Granovetter, 1978), o de los actores directamente involucrados (Donoso, 2013; Glasius y Pleyers, 2013; Von Büllow, 2009), existe menos evidencia respecto a quienes pueden apoyar la protesta, pero no necesariamente participar en ella. Esta es una pregunta relevante, porque una protesta no necesariamente puede entenderse solamente por quienes están directamente involucrados (como tiende a ser estudiado desde la perspectiva de los movimientos sociales) sino también por quienes directa o indirectamente, están conectados (en términos personales, sociales, emocionales o institucionales) con estos fenómenos. Tal como indican Kelly y Kelly (1994), la disposición a protestar refleja la identificación de los sujetos con los contextos sociopolíticos existentes y con los grupos contenciosos. De esta manera, el análisis de la disposición (a diferencia de los estudios sobre participación) entrega información sobre las percepciones y preferencias individuales que tienen las personas respecto de las acciones colectivas y la realidad social en la que viven (Veenstra y Haslam, 2000).

Considerando todo lo anterior, el artículo busca indagar respecto a qué lleva a los latinoamericanos a estar dispuestos a participar en una protesta, en un contexto marcado por importantes avances en materia económica y política. Para desarrollar este objetivo, utilizaremos datos de la Encuesta Latinobarómetro 2015 (Latinobarómetro, 2015). Si bien esta no es la única fuente de información aplicada a distintos países de la región que considera preguntas como las indagadas en este trabajo, sí es el que se ha aplicado desde hace más tiempo en la región y al mismo tiempo, el que tiene disponible datos de más reciente aplicación, lo que fue 
un criterio a considerar en la revisión y selección de preguntas que se transforman en las variables consideradas en este trabajo ${ }^{7}$, las que buscarán dar cuenta de los elementos planteados en esta sección.

\section{Variables}

La variable dependiente es la disposición a participar en una protesta, para lo que nos basamos en la pregunta respecto a en las que concurriría a protestar de una manera convencional ${ }^{8}$. Considerando nuestro objetivo de investigación, se construyó una variable dicotómica de tipo dummy, donde quienes han protestado o lo harían (de una manera convencional) son asignados con un valor $=1$, mientras quienes no protestarían bajo ninguna circunstancia tienen un valor $=0$.

Las variables independientes las clasificamos en torno a cuatro grupos, que recogen distintas discusiones conceptuales. En el primero de ellos, denominado Uso de Tecnología, analizamos preguntas relacionadas con el uso de herramientas tecnológicas, donde un mayor uso de la aplicación Twitter tendría un efecto de mayor disposición a protestar, al igual que una mayor exposición a Internet ${ }^{9}$. Si bien investigación relacionada con el uso de tecnologías en general ha indicado que este es un elemento más bien de difusión que de construcción de opinión pública (González - Bustamante, 2015), incluimos este set de variables debido a que ha sido

7. Un ejemplo son los estudios realizados en el marco de Latin American Publi Opinion Project o LAPOP, siendo una muestra de ello el realizado por Mason Moseley (2015). En nuestro caso, se optó por utilizar Latinobarómetro debido a que es el más reciente, al ser aplicado en 2015. El último disponible en LAPOP, en cambio, corresponde a 2014. Al respecto ver http://www. vanderbilt.edu/lapop/ y www.latinobarometro.org. [Fecha de acceso, 11 de septiembre de 2017].

8. El texto original de la pregunta es el siguiente: "Le voy a leer algunas acciones que la gente puede realizar y quiero que me diga si ha realizado alguna de ellas (1), si las podría realizar (2) o si nunca las haría bajo ninguna circunstancia (3): Asistir a manifestaciones autorizadas, protestas, marchas"... Como se puede observar, el fraseo de la pregunta considera tácticas de protesta convencionales (en el sentido de tradicionales), pero es un tanto vaga respecto de la legalidad (o ilegalidad) de estas acciones. Somma y Medel han mostrado que, para el caso chileno, la participación en protestas puede diferir respecto de su carácter (Somma y Medel, 2017).

9. En el caso del uso de Twitter, la pregunta original es la siguiente: ¿Usa Ud. alguno de los siguientes servicios de redes sociales si es que Ud. usa alguno? (Twitter). Respecto del uso de Internet, el texto original es el siguiente: ¿Alguna vez en su vida ha usado Ud. correo electrónico o se ha conectado a Internet? Las alternativas son: (1) Sí, todos los días. (2) Sí, ocasionalmente. (3) Sí, casi nunca. (4) No, nunca. En ambos casos, las respuestas "no sabe" y "no responde" se optó por considerarlas como valores perdidos. 
indicado como un elemento que facilitaría la movilización en una protesta (Castells, 2012; Glasius y Pleyers, 2013) o que el uso de medios sociales disminuiría la brecha socioeconómica, de edad y de género en la participación política (Valenzuela, Somma, Sherman y Arriagada, 2016).

El segundo grupo de variables considera preguntas respecto a la Valoración de la Democracia. En este caso, utilizamos una variable para identificar si el entrevistado considera que la democracia es el mejor sistema de gobierno pese a sus problemas; y la valoración de las personas respecto del grado que esta presenta en su respectivo país, que es medida en una escala de 1 a 10, donde el primer valor es nada y totalmente democrático, respectivamente ${ }^{10}$. El efecto que esperaríamos es que una mayor valoración de la democracia como sistema de gobierno tendería a una menor disposición a protestar (Schatzman, 2005; Moseley, 2015), y en ese sentido, una peor valoración del encuestado respecto al grado de la democracia en su país, implicaría una mayor probabilidad en la disposición a protestar, buscando, así, poner en tensión la tesis de Bellinger \& Arce (2011).

Dado que es una relación no lineal (Booth y Seligson, 2009), intentaremos analizarla, partiendo de la idea que, en las democracias, los ciudadanos con bajo apoyo a estos sistemas pueden y deben trabajar para lograr cambios dentro del sistema a través (aunque no únicamente) de una mayor disposición a las protestas. Adicionalmente, también, incluimos una variable para medir la percepción en la distribución del ingreso, donde esperaríamos que una mejor opinión respecto de este tema disminuyese la disposición a protestar ${ }^{11}$, en línea con lo planteado (para el caso de acciones de protesta) por Ortiz, Burke, Berrada, y Cortés (2013).

El tercer grupo de variables agrupa variables relacionadas con el Ejercicio de la Ciudadanía del encuestado. Para ello consideramos dos preguntas. La primera es el sufragio en la elección anterior, mientras que la segunda es el autoposicionamiento del ciudadano en la escala izquierda-derecha ${ }^{12}$. Esperaríamos que ir a

10. En el primer caso la pregunta original es la siguiente: “¿Está Ud. muy de acuerdo (1), de acuerdo (2), en desacuerdo (3) o muy en desacuerdo con las siguientes preguntas (4): La democracia puede tener problemas, pero es el mejor sistema de gobierno”. Respecto del grado de democracia, la pregunta original es la siguiente: "Con una escala de 1 a 10, le pedimos evaluar cuán democrático es (país). El "1" quiere decir que “(país) no es democrático” y el "10” quiere decir que “(país) es totalmente democrático” ¿Dónde pondría Ud. a (país)?”.

11. La pregunta original es la siguiente: “¿Cuán justa cree Ud. que es la distribución del ingreso en (país)?” (P18St en el cuestionario), siendo sus alternativas muy justa (1), justa (2), injusta (3) y muy injusta (4).

12. La pregunta original que indaga en el recuerdo de voto es la siguiente: "Con respecto a 
votar (dado que implica un mayor ejercicio de la ciudadanía) implicaría una mayor disposición a protestar. De esta forma, entenderemos las distintas formas de participación política (voto y protesta) como formas complementarias, más que como formas contradictorias (Ekman y Amna, 2012), siguiendo la evidencia de estudios anteriores, que han mostrado que los latinoamericanos más politizados tienden a participar más en protestas (Moseley y Moreno, 2010). Respecto de la identificación política, el efecto esperado es que una mayor identificación con la izquierda -que en las protestas aparece como un sector con una mayor participación en este tipo de eventos en la región-, en línea con lo mostrado por Arce (2010), implicaría un aumento en la probabilidad de estar dispuesto a protestar.

Finalmente, en el cuarto grupo, analizamos variables sociodemográficas, a fin de caracterizar a los encuestados. En el caso del género del encuestado, asumimos un efecto mayor en el caso de los hombres respecto de las mujeres, mientras que respecto de la edad, se esperaría que los más jóvenes tuviesen una mayor probabilidad de estar dispuestos a protestar, tal como han discutido recientes estudios (Coe y Vandegrift, 2015; Treviño, Béjares, Villalobos y Naranjo, 2017).

Una síntesis de las variables independientes consideradas, de cómo estas fueron operacionalizadas y el efecto que se esperaría de cada una de ellas sobre la variable dependiente se detalla en el Anexo 1.

\section{Método}

En primer lugar, se genera una descripción de los resultados de las variables dependiente e independiente. Posteriormente analizamos la probabilidad que el encuestado tenga disposición a protestar. Para ello empleamos regresión logística, que es la técnica apropiada para variables categóricas binarias (Hosmer, Stanley y Sturdivant, 2013; Long y Freese, 2014; Faraway, 2017). Esta estimación se efectuó en dos pasos. En primer lugar, se realizan cuatro modelos de regresión logística, donde cada uno de ellos representa a los respectivos grupos de variables de manera diferenciada. En segundo lugar, y considerando los resultados de la etapa previa, seleccionamos

la última elección presidencial, ¿Qué hizo Ud.?” Las alternativas fueron las siguientes: Voté en la última elección (1); Decidí no votar en la última elección (2); Me impidieron votar en la última elección (3); No tuve tiempo para votar (4); No voté por otras razones (5); No estaba registrado para votar (6); No recuerdo lo que hice/no sé (7). Para el caso del autoposicionamiento en la escala izquierda-derecha, el texto de la pregunta es el siguiente: "En política se habla normalmente de "izquierda" y "derecha”. En una escala dónde “0” es la "izquierda” y "10" la "derecha”, ¿dónde se ubicaría Ud.?”. 
variables de acuerdo a la confiabilidad de sus parámetros o a la relevancia de su inclusión en términos teóricos, las que posteriormente son evaluadas de manera conjunta en dos modelos agregados.

Para efectos de interpretación de resultados de los modelos, cabe señalar que lo más intuitivo es interpretar el signo del parámetro que su valor. El parámetro corresponde al Logartitmo de Odd, donde en el caso de ser positivo, indica que esa variable tiene como efecto incrementar la probabilidad de ocurrencia de la dependiente, en este caso la probabilidad de una mayor disposición a protestar. Junto a este parámetro, entre paréntesis se indica su error estándar. A fin de facilitar la presentación de resultados, se exponen la estimación de las respectivas probabilidades predichas. La probabilidad se estima en un rango que oscila entre un valor mínimo de 0 , que es la ausencia de la misma, y un máximo de 1 , que sería una probabilidad del $100 \%$. La estimación de probabilidades fue hecha a partir de su media mediante el comando "margins", disponible a partir de Stata 12.

\section{Resultados}

En general los latinoamericanos no muestran una disposición muy elevada a participar en actos de protesta, con EL $62 \%$ de las menciones que indican que no participaría, frente a un $38 \%$ que lo ha hecho o que estaría dispuesto a hacerlo. En relación a las variables independientes consideradas, tanto el grado de democracia como la escala izquierda-derecha se ubican en torno a la media, lo que significa que la valoración de la democracia en su respectivo país tiende a ser medianamente democrático, donde sus ciudadanos tenderían a ubicarse políticamente en el centro. En el caso de esta última, presenta el menor número de observaciones porque se excluyen las alternativas que no indican identificación, pues tendrían un efecto de sesgar la media de la escala hacia la izquierda como consecuencia del valor asignado a estas respuestas. La edad promedio de los encuestados es de 40 años.

Para el caso de las variables categóricas, se considera como descriptivo la moda en vez de la media. Al respecto, se puede indicar que los encuestados utilizan escasamente la red social Twitter y en general están menos expuestos a Internet. Asimismo, consideran que la democracia es el mejor sistema de gobierno, señalan haber votado en la última elección y perciben que la distribución del ingreso es injusta. Al mismo tiempo, el porcentaje de mujeres es levemente superior. Una síntesis de las variables explicativas se sintetiza en el Cuadro 2. 


\section{Cuadro 2.}

Estadísticos descriptivos de las variables

\begin{tabular}{|c|c|c|c|c|c|c|}
\hline Variable & Media & $\begin{array}{l}\text { Desviación } \\
\text { Estándar }\end{array}$ & Min. & Máx. & Moda & $\mathrm{N}$ \\
\hline $\begin{array}{l}\text { Grado de } \\
\text { Democracia }\end{array}$ & 5,85 & 2,59 & $\begin{array}{l}1 \\
\text { (No democrático) }\end{array}$ & $\begin{array}{l}10 \\
\text { (Democrático) }\end{array}$ & -- & 19.089 \\
\hline $\begin{array}{l}\text { Escala Izquierda } \\
\text { Derecha }\end{array}$ & 5,39 & 2,98 & $\begin{array}{l}0 \\
\text { (Izquierda) }\end{array}$ & $\begin{array}{l}10 \\
\text { (Derecha) }\end{array}$ & -- & 15.889 \\
\hline Edad & 40,37 & 16,49 & 16 & 98 & -- & 20.250 \\
\hline Uso de Twitter & -- & -- & $\begin{array}{l}0 \\
\text { (No menciona) }\end{array}$ & $\begin{array}{l}1 \\
\text { (Menciona) }\end{array}$ & $\begin{array}{l}0 \\
(88 \%)\end{array}$ & 19.050 \\
\hline Uso de Internet & & & $\begin{array}{l}1 \\
\text { (Mucho) }\end{array}$ & $\begin{array}{l}3 \\
\text { (Nada) }\end{array}$ & $\begin{array}{l}3 \\
(47 \%)\end{array}$ & 18.831 \\
\hline $\begin{array}{l}\text { Democracia es el } \\
\text { mejor sistema de } \\
\text { gobierno }\end{array}$ & -- & -- & 1 (De acuerdo) & $\begin{array}{l}3 \\
(\mathrm{NS} / \mathrm{NR})\end{array}$ & $\begin{array}{l}1 \\
(72 \%)\end{array}$ & 20.250 \\
\hline $\begin{array}{l}\text { Percepción de la } \\
\text { Distribución del } \\
\text { ingreso }\end{array}$ & -- & -- & $\begin{array}{l}1 \\
\text { (Justa) }\end{array}$ & 2 & $2(75 \%)$ & 19.176 \\
\hline $\begin{array}{l}\text { Voto en la } \\
\text { última elección }\end{array}$ & -- & -- & $\begin{array}{l}0 \\
(\mathrm{No})\end{array}$ & $\begin{array}{l}1 \\
\text { (Sí) }\end{array}$ & $1(81 \%)$ & 18.737 \\
\hline Género & -- & -- & $\begin{array}{l}1 \\
\text { (Hombres) }\end{array}$ & $\begin{array}{l}2 \\
\text { (Mujeres) }\end{array}$ & $2(52 \%)$ & \\
\hline
\end{tabular}

Fuente: Latinobarómetro 2015.

Ahora bien, respecto de los modelos de regresión estimados, una primera consideración es que las pruebas de bondad de ajuste en los modelos son bajas, aun cuando en general en este tipo de modelos ello no es un problema serio. En términos de las medidas de bondad de ajuste, el mejor se produce en el M3, al tomar en cuenta el ajuste por el criterio de información bayesiano (BIC), que es el menor valor. La inclusión del pseudo $\mathrm{R}^{2}$ de Mac-Fadden se presenta a fin de facilitar la interpretación de resultados, aún esta lectura debe ser tomada con cuidado, pues no es un modelo basado en mínimos cuadrados ordinarios.

En estos, si bien el mejor ajuste se produce en el Modelo 3 (valor de Criterio de Información Bayesiano o BIC más bajo), al mismo tiempo presenta la menor cantidad de observaciones, situación que no debiera ser problemática considerando que el número de casos sigue siendo importante (15.898). 
En relación a los parámetros obtenidos, en las variables relacionadas con uso de herramientas tecnológicas (M1) se muestra que el empleo de estas implica una mayor disposición a involucrarse en algún tipo de protesta convencional. Este efecto es más importante entre quienes están más expuestos a Internet, y en menor medida entre quienes utilizan la red social Twitter (Cuadro 3), en la misma línea con lo encontrado por otros autores (Valenzuela et al, 2015). De esta manera, la participación en redes sociales no solo actuaría como un factor que permitiría las conexiones y relaciones entre protestantes, sino que también aumentaría la disposición a protestar, probablemente debido a la mayor exposición sobre las demandas implicadas en las acciones colectivas (González - Bustamante, 2015).

Respecto a una mayor valoración de la democracia, quienes tienen una mejor opinión de esta como sistema de gobierno presentan una mayor disposición a la protesta respecto a quienes la valoran poco o son indiferentes, en la misma línea a lo encontrado por Moseley (2015). Estos resultados se complementan respecto a su grado de funcionamiento, que implica que a medida que esta mejora, disminuye una mayor disposición a protestar, aunque su nivel de significancia es bajo. Ello mostraría que la crítica a la democracia no estaría influenciando en la disposición a protestar, aunque eventualmente sí una menor valoración respecto de su funcionamiento. Tal como indican Booth y Selington (2009) esto podría deberse a que la relación entre valoración de la democracia y acción colectiva no es lineal, sino que presenta una forma de "U", donde altas y bajas valoraciones de la democracia se relacionan con mayores disposiciones a la protesta.

Un resultado que es altamente llamativo es que una evaluación crítica hacia la distribución del ingreso influye poco o nada en la disposición a protestar, pues si bien quienes creen que un reparto desigual de la riqueza es injusto tienen una menor probabilidad de estar dispuestos a protestar, este resultado no es significativo. De esta manera, no encontramos que la percepción sobre la justicia distributiva sea un factor explicativo relevante para entender la disposición a la acción colectiva, lo que, a lo menos en parte, se contradice con lo planteado por Castillo, Palacios, Joignant y Tham (2015) para el caso chileno y que se alinean más con evidencia comparada, que ha puesto en tela de juicio esta relación (Poulos, 2012).

Respecto a una mayor participación en política (M3), quienes votaron en la última elección presidencial, aumentan de manera importante su disposición a protestar que quienes no ejercieron este derecho. De esta manera, parece ser que, en línea de lo planteado por Ekman y Amna (2012), las distintas formas de participación cívica (en este caso, más o menos institucionales) no competirían entre sí, 
sino que se entenderían como dos formas complementarias, pudiendo entender el proceso de acción colectiva como un proceso de profundización de la democracia más que como una forma de crítica a esta (Rosanvallon, 2008).

$\mathrm{Al}$ mismo tiempo, los encuestados que se identifican más con la izquierda tienen una mayor probabilidad de estar dispuestos a protestar, lo que es consistente con lo esperado históricamente, pero es especialmente interesante en el contexto de un retroceso o, a lo menos, estancamiento, del llamado "ciclo progresista" o "ciclo post neoliberal", que tuvo su auge durante la primera década del siglo XXI (Sader, 2008; Moreira, 2017). Finalmente, al tomar en cuenta el género y edad de los entrevistados (M4), se confirman los supuestos que se plantean en el análisis descriptivo, pues los hombres tienen una mayor probabilidad que las mujeres de estar dispuestos a protestar, al igual que los más jóvenes, lo que se encuentra en la misma línea a lo encontrado por Moseley (2015) al utilizar la Encuesta LAPOP entre 2008 y 2012, lo que podría estar dando cuenta de una cierta estabilidad en este conjunto de variables.

\section{Cuadro 3.}

Variable dependiente: Disposición a protestar $=1$

\begin{tabular}{|c|c|c|c|c|}
\hline & M1 & M2 & M3 & M4 \\
\hline Usa Twitter $(=1)$ & $\begin{array}{l}0,0337 \\
(0,0490)\end{array}$ & -- & -- & -- \\
\hline Usa poco Internet (Referencia mucho) & $\begin{array}{l}-0,229 * * * \\
(0,0639)\end{array}$ & -- & -- & -- \\
\hline No usa Internet (Referencia mucho) & $\begin{array}{l}-0,693 * * \\
(0,0337)\end{array}$ & -- & -- & -- \\
\hline $\begin{array}{l}\text { Democracia no es el mejor sistema } \\
\text { (Referencia es el mejor) }\end{array}$ & -- & $\begin{array}{l}-0,392 * * * \\
(0,0385)\end{array}$ & -- & -- \\
\hline NS/NR (Referencia es el mejor) & -- & $\begin{array}{l}-0,573 * * * \\
(0,0932)\end{array}$ & -- & -- \\
\hline $\begin{array}{l}\text { Grado de democracia ( } 1=\text { No democrático; } \\
10=\text { Totalmente democrático) }\end{array}$ & -- & $\begin{array}{l}-0,00209 \\
(0,00618)\end{array}$ & -- & -- \\
\hline $\begin{array}{l}\text { Distribución injusta del ingreso } \\
\text { (Referencia 1=Justa) }\end{array}$ & -- & $\begin{array}{l}-0,0174 \\
(0,0360)\end{array}$ & -- & -- \\
\hline Voto en la última elección Referencia (1=Sí) & -- & -- & $\begin{array}{l}0,264 * * * \\
(0,0459)\end{array}$ & -- \\
\hline $\begin{array}{l}\text { Escala Izquierda - Derecha (0 Izquierda - } \\
10 \text { Derecha) }\end{array}$ & -- & -- & $\begin{array}{l}-0,0496 * * \\
(0,00569)\end{array}$ & -- \\
\hline
\end{tabular}




\begin{tabular}{lllll}
\hline Género (Referencia Hombres) & - & - & - & $\begin{array}{l}-0,214 * * * \\
(0,0293)\end{array}$ \\
\hline Edad & - & - & -- & $\begin{array}{l}-0,0131 * * * \\
(0,000909)\end{array}$ \\
\hline Constante & & & & $-0,139 * * *$ \\
& $(0,0244)$ & $\begin{array}{l}-0,304 * * * \\
(0,0536)\end{array}$ & $\begin{array}{l}-0,352 * * * \\
(0,0517)\end{array}$ & $\begin{array}{l}0,163 * * * \\
(0,0416)\end{array}$ \\
\hline $\mathrm{R} 2($ MFadj) & 0,020 & 0,005 & 0,005 & 0,010 \\
\hline $\mathrm{BIC}$ & $-471,452$ & $-100,066$ & $-87,613$ & $-246,668$ \\
\hline $\mathrm{N}$ & 18.559 & 18.102 & 14.625 & 19.959 \\
\hline$* \mathrm{p}<0.05, * \mathrm{p}<0.01, * * \mathrm{p}<0.001$ & & & \\
\hline
\end{tabular}

A partir de estos resultados, se consideraron las variables que resultaron más significativas en cada uno de los grupos de variables para ser analizadas en un modelo conjunto (M5). Asimismo, se reevalúa el grado de democracia mediante una interacción con el voto en la elección anterior, incorporándolo en un sexto modelo (M6), porque el efecto que puede tomar la valoración del funcionamiento del sistema democrático puede estar dependiendo de si participan o no en este mediante el voto. En ese sentido, supondríamos que en el caso que los ciudadanos no participen, una peor valoración de la democracia debiera aumentar su disposición a protestar.

Los resultados (Cuadro 4) confirman, en general, los resultados previos, aunque el principal cambio que se puede obtener del modelo 6 (M6), es que la inclusión del efecto mediador del voto en la última elección, aumentó el efecto de la valoración del grado de democracia sobre una mayor disposición a protestar de manera bidireccional. Así, cuando los ciudadanos no participaron en la última elección, una menor valoración del funcionamiento de la democracia aumentó la disposición a protestar $(-0,031)$. Pero al mismo tiempo, cuando los ciudadanos ejercen su derecho cívico, una evaluación positiva del estado del sistema democrático, también aumenta una mayor disposición a protestar. Esto tiende a reforzar la idea planteada por Moseley (2015), en el sentido de que es la combinación entre involucramiento cívico y funcionamiento democrático deficiente (o la percepción de este, en nuestro caso) lo que genera una mayor disposición hacia la acción colectiva. 


\section{Variable dependiente: Disposición a protestar $=1$}

\begin{tabular}{|c|c|c|}
\hline & M5 & M6 \\
\hline Usa poco Internet (Referencia mucho) & $\begin{array}{l}-0,159 \\
(0,0858)\end{array}$ & $\begin{array}{l}-0,0147^{*} \\
(0.073)\end{array}$ \\
\hline No usa Internet (Referencia mucho) & $\begin{array}{l}-0,551 * * * \\
(0,0347)\end{array}$ & $\begin{array}{l}-0,545^{* * *} \\
(0,039)\end{array}$ \\
\hline Democracia no es el mejor sistema (Referencia es el mejor) & $\begin{array}{l}-0,387 * * * \\
(0,0472)\end{array}$ & $\begin{array}{l}-0,386^{* * *} \\
(0,046)\end{array}$ \\
\hline NS/NR (Referencia es el mejor) & $\begin{array}{l}-0,355^{* *} \\
(0,120)\end{array}$ & $\begin{array}{l}-0,320 * * * \\
(0,137)\end{array}$ \\
\hline $\begin{array}{l}\text { Grado de democracia } \\
(1=\text { No democrático; } 10=\text { Totalmente democrático })\end{array}$ & -- & $\begin{array}{l}-0,031 * * \\
(0,010)\end{array}$ \\
\hline Grado de Democracia* Voto & -- & $\begin{array}{l}0,043 * * \\
(0,008)\end{array}$ \\
\hline Voto en la última elección Referencia (1=Sí) & $\begin{array}{l}0,267 * * * \\
(0,0526)\end{array}$ & -- \\
\hline Escala Izquierda - Derecha (0 Izquierda - 10 Derecha) & $\begin{array}{l}-0,0446 * * * \\
(0,00621)\end{array}$ & $\begin{array}{l}-0,044 * * * \\
(0.004)\end{array}$ \\
\hline Hombres & $\begin{array}{l}0,202 * * * \\
(0,0342)\end{array}$ & $\begin{array}{l}0,191 * * * \\
0,033\end{array}$ \\
\hline Edad & $\begin{array}{l}-0,00532 * * * \\
(0,00137)\end{array}$ & $\begin{array}{l}-0,005^{* * *} \\
(0,001)\end{array}$ \\
\hline Constante & $\begin{array}{l}0,0838 \\
(0,0720)\end{array}$ & $\begin{array}{l}0,667 * * * \\
(0,092)\end{array}$ \\
\hline R2 (MFadj) & 0,03 & 0,03 \\
\hline BIC & $-473,430$ & $-431,257$ \\
\hline $\mathrm{N}$ & 13.556 & 13.165 \\
\hline
\end{tabular}

Ahora bien, la estimación de las probabilidades predichas para las variables categóricas nos indican que si bien esas tienen alguna capacidad predictiva, esta es más bien baja. Aun así, existe una cierta heterogeneidad entre las variables analizadas. Como se puede observar en el Anexo 2, las diferencias son mayores entre los usuarios de Internet, de acuerdo a quienes tienen una mayor valoración de la democracia y a si participaron en la última elección. 
En el caso de las variables escalares (identificación izquierda - derecha, edad y grado de democracia), se utiliza como categoría de comparación el recuerdo de voto en la elección pasada, debido al efecto mediador que presentó en el M6 respecto al grado de democracia, porque mostraría algunas orientaciones respecto a cómo evolucionarían estas otras dos variables, y donde la inclusión del voto como un mediador del efecto del mayor grado de valoración de la democracia sobre la disposición a protestar muestra que las principales diferencias se van produciendo a medida hay una mayor valoración del funcionamiento de la democracia. De esta manera, entre quienes votan y tienen una valoración máxima de esta, la voluntad de involucrarse en una protesta llega a un $42 \%$, frente a un $32 \%$ de quienes, pese a calificar el sistema con la nota máxima (10) no votaron (Gráfico 1).

\section{Gráfico 1.}

Disposición a la protesta y democracia

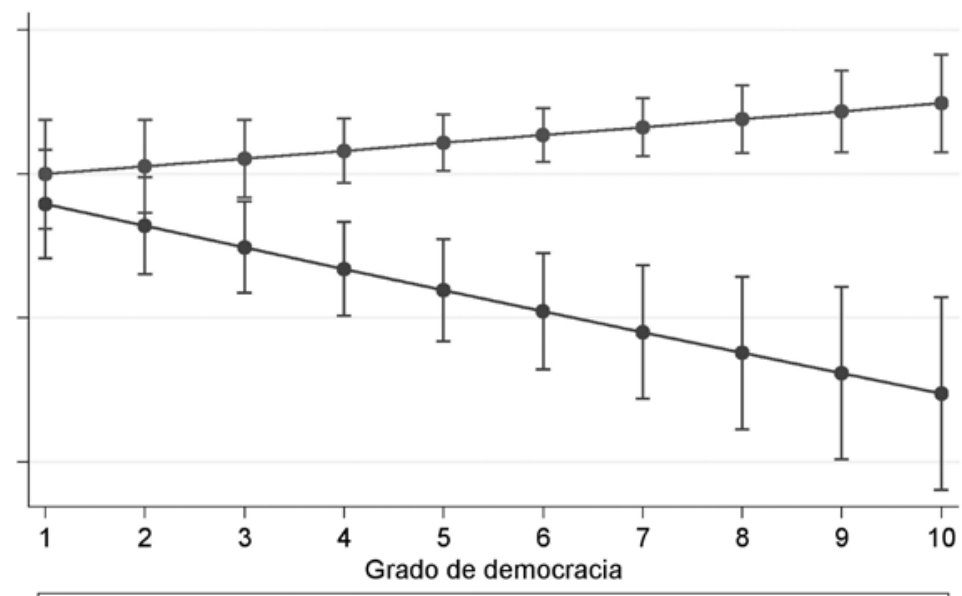

No votó en la última elección

Votó en la última elección

Fuente: Latinobarómetro 2015.

Finalmente, en el caso de la estimación por países, a partir de los resultados del modelo 6 nos indican que este modelo tiene una mayor probabilidad de predecir la disposición a la protesta para los casos de Chile y Brasil, que son precisamente los países donde se registraron las protestas con mayor masividad en esos años (Somma y Medel, 2017; Bringel, 2016) seguidos de Paraguay y Colombia. En los casos chileno y brasileño, este modelo estima una probabilidad del $93 \%$ y $85 \%$, que 
equivalen a 3.9 y 3.4 veces la probabilidad del promedio de LAC $^{13}$. En términos de la bondad de ajuste del modelo, el indicador más fiable es la mejora al incluir las variables respecto de la estimación de un modelo nulo (Likelihood Ratio Test ${ }^{14}$ ), donde un mayor valor indica un mayor incremento en la mejoría (Cuadro 5).

\section{Cuadro 5.}

Probabilidad (Pr) por países (M6), estimada por conjunto de valores

\begin{tabular}{|c|c|c|c|c|c|}
\hline & $\mathrm{N}$ & LRT & Pseudo $\mathrm{R}^{2}$ & Probabilidad & $\begin{array}{l}\text { Diferencia respecto } \\
\text { a } \operatorname{Pr}(\mathrm{LAC})\end{array}$ \\
\hline Argentina & 876 & 51,23 & 0,0441 & 0,12 & 0,50 \\
\hline Bolivia & 732 & 12,17 & 0,0121 & 0,17 & 0,72 \\
\hline Brasil & 611 & 59,16 & 0,0805 & 0,83 & 3,44 \\
\hline Chile & 741 & 95,73 & 0,1065 & 0,93 & 3,89 \\
\hline Colombia & 891 & 63,20 & 0,0539 & 0,67 & 2,78 \\
\hline Costa Rica & 799 & 55,82 & 0,0575 & 0,31 & 1,29 \\
\hline República Dominicana & 752 & 28,70 & 0,0289 & 0,22 & 0,91 \\
\hline Ecuador & 828 & 35,28 & 0,0382 & 0,21 & 0,87 \\
\hline El Salvador & 725 & 22,52 & 0,0320 & 0,10 & 0,43 \\
\hline Guatemala & 592 & 22,10 & 0,0369 & 0,13 & 0,53 \\
\hline Honduras & 741 & 20,93 & 0,0243 & 0,15 & 0,64 \\
\hline Nicaragua & 629 & 32,27 & 0,0432 & 0,12 & 0,49 \\
\hline Panamá & 753 & 25,83 & 0,0286 & 0,12 & 0,52 \\
\hline Paraguay & 639 & 33,00 & 0,0417 & 0,77 & 3,23 \\
\hline Perú & 825 & 29,75 & 0,0269 & 0,33 & 1,37 \\
\hline Uruguay & 1.036 & 150,06 & 0,1169 & 0,11 & 0,46 \\
\hline Venezuela & 989 & 38,07 & 0,0333 & 0,24 & 0,99 \\
\hline América Latina & 13.165 & 516,63 & 0,0299 & 0,24 & 1,00 \\
\hline
\end{tabular}

13. Este resultado se interpreta a partir de $R=\frac{\mathrm{PPP}_{\mathrm{P}}}{\mathrm{P}_{\Gamma^{\prime}}}$ del ratio $(\mathrm{R})$ entre la probabilidad para cada país $\left(\operatorname{Pr}_{\mathrm{p}}\right)$ respecto al conjunto de América Latina $\left(\operatorname{Pr}_{\mathrm{A}}\right)$. En el caso de Chile el resultado de $3.9=0.93 / 0.24$

14. Esta es una de las medidas de ajuste para modelos de máxima verosimilitud, como el empleado en este trabajo, que se expresa en la fórmula $-2(L L n-L L f)$. $L L n c$ orresponde al logaritmo de máxima verosimilitud de un modelo sin co-variables o nulo, $L L f$ expresa este resultado con la inclusión de las mismas y -2 indica dos veces las diferencia entre ambas (Long y Freese, 2014). 


\section{Conclusiones}

El objetivo de la investigación fue explorar los factores que podrían estar explicando la disposición de los latinoamericanos a protestar, en un contexto general de crecimiento económico y (lenta) consolidación de la democracia. Vistos en su conjunto, los resultados muestran que supuestos importantes no se estarían cumpliendo y, lo que es más llamativo, evidencian paradojas y potenciales preguntas a ser exploradas.

En primer lugar, variables clásicas utilizadas en los estudios de protestas (el uso de redes sociales o la satisfacción con la democracia o la percepción de las injusticias en materIa de ingreso) no parecen ser especialmente relevantes para explicar esta disposición. De modo hipotético, esto podría estar mostrando la distancia existente entre la disposición y la acción, aspecto central para el entendimiento complejo de las protestas (Van Stekelenburg y Klandermans, 2013) y las condiciones particulares en las que se desarrolla esta ola de protestas.

En segundo término, aparece como un importante factor explicativo el papel que juega la participación de la democracia y la valoración de esta. Por un lado, porque el ejercicio del voto aparece como uno de los parámetros más eficientes, pero principalmente por el efecto mediador que tiene en variables como el grado de democracia y en acentuar la importancia de una identificación con la izquierda y, en menor medida, con la edad de los encuestados. Adicionalmente, se muestra que una mayor valoración de la democracia implica una mayor disposición a involucrarse en alguna de estas acciones, lo que puede ser entendido como que la protesta es entendida por los sujetos como un elemento profundizador (más que un debilitador) de la democracia. En un contexto donde el "fin del ciclo progresista" (Moreira, 2017) parece como una realidad histórica, considerar estos factores como detonantes de la disposición a la protesta puede permitir profundizar en la relación entre democracia, política y acción colectiva y sobre las características de la nueva ola de protestas (Roberts, 2017).

Una tercera conclusión tiene que ver con la variabilidad que se observa al estimar las probabilidades de disposición segmentadas por países. El modelo planteado tras distintas pruebas, ajusta de buena manera principalmente para los países que han enfrentado protestas masivas en estos años como Chile, Brasil y en menor medida Colombia, pero no es efectivo para países que presentan una historia más amplia en movilizaciones de este tipo de acciones, como Argentina. Ello plantea importantes preguntas respecto de diferencias estructurales entre los países sobre las variables relevantes para entender la disposición a la protesta, cuestionando así el entendimiento de América Latina y el Caribe como un conjunto compacto 
respecto de este fenómeno. Como ha indicado Cortés (2017) para el caso chileno, es posible pensar la existencia de sincronías y asincronías entre los países de la región, poniendo en tensión análisis comparativos en un mismo momento, como el realizado en este caso.

Junto con entregar algunas luces sobre la dinámica de las protestas, sus factores influyentes y su relación con la política latinoamericana, el artículo también produce una serie de desafíos para próximas investigaciones. En términos metodológicos, los resultados dan cuenta de la dificultad de realizar estimaciones precisas respecto a fenómenos que responden a percepciones y a conceptos que están latentes. Una aproximación posible sería el uso de regresiones que permitan trabajar con variables sin necesidad de dicotomizarlas y con ello, evitar cierto riesgo de perder información mediante la recodificación de manera binaria, como sería el caso de los modelos multinomiales. No obstante, el riesgo sería perder cierta capacidad predictiva al establecer múltiples categorías de comparación. Al mismo tiempo, se debiera avanzar hacia la estimación de un análisis segmentado por países, tomando en cuenta la variabilidad en los contextos nacionales, que es un aspecto que no es abordado por las características del análisis que es de corte transversal.

Un segundo problema tuvo que ver con la identificación de variables que permitirían dar cuenta de una manera precisa de aspectos tradicionalmente considerados como factores relevantes para el ejercicio de la protesta, como el nivel socioeconómico y el nivel educativo de los encuestados y que pudieran ser incluidas en los modelos. Si bien en la encuestas se incluyen preguntas de este tipo, en general estas cuentan con una percepción subjetiva, en especial respecto del ingreso, por lo que no pareció adecuada su inclusión en este caso. Dada la relevancia que tienen estos aspectos en la literatura sobre protesta (Scholzman, Verba y Brady, 1999; Marien, Hooghe y Quintelier, 2010; Klesner, 2007) este es un elemento que debe ser contrastado en futuras investigaciones, usando otras fuentes de información.

\section{Referencias bibliográficas}

AlmeIDA, P. D. (2007). Defensive Mobilization: Popular Movements against Economic Adjustment Policies in Latin America. Latin American Perspectives (34) (3), 123-139.

Altman, D., \& Pérez-LiÑán, A. (2002). Assessing the Quality of Democracy: Freedom, Competitiveness and Participation. Democratization (9)(2), 85-100. 
Arce, M. (2010). Parties ans social protest in Latin America's Neoliberal Era. Party Politics (16)(5), 669-686.

Bellinger, P. T., \& Arce, M. (2011). Protest and Democracy in Latin America's Market Era. Political Research Quarterly (64)(3), 688-704.

Bertelsmann Stiftung. (2017). Transformation Index BTI 2016. Regional Report Latin America.Abgerufen am 14. Septiembre 2017 von https://www.btiproject.org/en/reports/regional-reports/latin-america-and-the-caribbean/

Boоth, J., \& Seligson, M. A. (April 2,4, 2009). Legitimacy and political participation in eight latin american nations. Paper presentado en Midwest Political Science Meeting, (S. 44). Chicago.

Bringel, B. (8. 12 2016). De las protestas de 2013 al golpe de 2016.Von Viento Sur: http://vientosur.info/spip.php?article11989 abgerufen

CAstells, M. (2012). Redes de indignación y esperanya. Madrid: Alianza.

Castillo, J. C., Palacios, D., Joignant, A., \& Tham, M. (2015). Inequality, Distributive Justice and Political Participation: An Analysis of the Case of Chile. Bull Latin Am Res (34)(4), 486-205.

CEPAL. (2017). Estudio Económico de América Latina y el Caribe: La Agenda 2030 para el Desarrollo Sostenible y los desafíos del financiamiento para el desarrollo. Santiago: CEPAL.

CEPAL. (2017). Panorama Social de América Latina 2016. Santiago: Naciones Unidas.

Coe, A., \& Vandegrift, D. (2015). Youth Politics and Culture in Contemporary Latin America: A review. Latin American Politics and Society (37)(2), 132-157.

CORTÉs, A. (2017). El contra-ciclo político chileno: asincronías y contra-tendencias frente a la política latinoamericana. Santiago: Universidad Alberto Hurtado.

Della Porta, D. (2008). Eventful protest, global conflicts. Conference of the Nordic Sociological Association. Aahurs.

Diamond, L., \& Morlino, L. (2005). Assesing the quality of democracy. Washington: Johns Hopkins University Press.

DiAni, M., \& Mische, A. (2015). Network approaches and social movements. In D. Della Porta, \& M. Diani, The Oxford Handbook of Social Movements (S. 326/339). New York: Oxford Universitz Press.

Doherty, B., Plows, A., \& Wall, D. (2007). Environmental direct action in Manchester, Oxford and North Wales: A protest event analysis. Environmental Politics (16) (5), 805-825.

Donoso, S. (2013). Dynamics of Change in Chile: Explaining the Emergence of the Pingüino Movement. Journal of Latin American Studies (45), 1-29. 
Eisenstadt, S. N. (1968). Moderinzación. Movimientos de protesta y cambio social. Buenos Aires: Amorrortú.

Ekman, J., \& Amna, E. (2012). Political participation and civic engagement. Human Affairs (22), 283-300.

Faraway, J. J. (2017). Extending the Linear Model with R. Generalized Linear, Mixed Effects and Nonparametric Regression Models. Chapman \& Hall/ CRC Texts in Statistical Science.

Finkel, S., \& Muller, E. (1998). Rational choice and the dynamic of collective political action. American Political Science Review (92)(1), 37-49.

Flam, H. (2015). Micromobilzations and emotions. In D. Della Porta, \& Diani, The Oxford Handbook of Social Movements (S. 326-339). New York: Oxford University Press.

Garretón, M. A. (2002). La transformación de la acción colectiva en América Latina. CEPAL (76), 7-25.

Glasius, M., \& Pleyers, G. (2013). The global moment of 2011: Democracy, social justice and dignity. Development and change (44) (3), 547-567.

GonZÁlez - Bustamante, B. (2015). Evaluando Twitter como indicador de opinión pública: una mirada al arribo de Bachelet a la presidencial chilena 2013. SAAP (9)(1), 119-141.

Goodwin, J., Jasper, J., \& Polleta, F. (2007). Emotional dimensions of social movements. In D. Snow, S. Soule, \& H. Kriesi, The Blackwell Companion of Social Movements. Malden: Blackwell Publishing.

Granovetter, M. (1978). Threshold Models of Collective Behavior. American Journal of Sociology (83)(6), 1420-1443.

Hosmer, D. W., S. L., \& Sturdivant, R. X. (2013). Applied Logistic Regression. New Jersey: Wiley.

Kelly, C., \& KelLy, J. (1994). Who gets involved in collective action?: Social psyhological determinants of individual participations in trade unions. Human Relations (47)(1), 63-88.

King, B. G., \& Pearce, N. A. (2010). The contentiousness of markets: Politics, social movements, and institutional change in markets. Annual Review of Sociology (36), 249-267.

Klandermans, B. (2007). The demand and supply of participation: Social-psychological correlates of participation in social movements. In D. A. Snow, S. A. Soule, \& H. Kriesi, The Blackwell Companion to Social Movements (S. 360-379). Blackwell Publishing.

Klesner, J. L. (2007). Social Capital and Political Participation in Latin America: 
Evidence from Argentina, Chile, Mexico, and Peru. Latin American Research Review (47)(2), 1-32.

Koopmans, R., \& Rucht, D. (2002). Protest Event Analysis. In B. Klandermans, \& S. Staggenborg, Methods of Social Movement Research (S. 231-259). Minneapollis: University of Minnesota Press.

Krugman, P. (2012). Acabad ya con esta crisis. Madrid: Crítica.

Latinobarómetro. (2015). Encuesta Latinobarómetro. Cuestionario 2015.

Liao, T. F. (1994). Interpreting Probability Models. Logit, Probit and Other Genralized Linear Models. California: SAGE.

Long, J. S., \& Freese, J. (2014). Regression Models for Categorical Dependent Variables Using Stata. Texas: Stata Corp.

Marien, S., Hooghe, M., \& Quintelier, E. (2010). Inequalities in Non-institutionalised Forms of Political Participation: A Multi-level Analysis of 25 countries. Political Studies (58)(1), 187-213.

Marshall, M. G. (2013). POLITY IV Project. Political Regime and Transitions, 1800-2013. Data User's Manual. Societal-Systems Research Inc.

Martínez, C. (2015 ). Presidential survival in South America: Rethinking the role of Democracy. International Political Science Review, 1-16.

Medel, R., \& Somma, N. (2016). ¿Marchas, ocupaciones o barricadas? Explorando los determinantes de las tácticas de la protesta en Chile. Política y gobierno, 163-199.

Moreira, C. (2017). El largo ciclo del progresismo latinoamericano y su freno: los cambios políticos en América Latina en la última década (2003-2015). Revista Brasileira de Ciencias Sociais (32)(93), 1-28.

Moseley, M. W. (2015). Contentious Engagement: Understanding Protest Participation in Latin American Democracies. Journal of Politics in Latin America (7) (3), 3-48.

Moseley, M., \& Moreno, D. (2010). The Normalization of Protest in Latin America. American Bar. Abgerufen am Noviembre 2017 von American Barometers Insights: 2010 (42): https://pdfs.semanticscholar.org/9442/4e4dec7f5b020 ecd1926b95c27a8c2e7d025.pdf

O’Donell, G. (1994). Delegative Democracy. Journal of Democracy (5)(1), 55-69.

OlzAK, S. (1989). Analysis of events in the study of collective action. Annual Review of Sociology (15), 119-141.

Ortiz, I., Burke, S., Berrada, M., \& Cortés, H. (2013). World Protest 206-2013. New York: Initiative for Policy Dialogue and Friedrich-Ebert-Stiftung New York. 
Pérez-Liñán, A. (2009). Juicio político al presidente y nueva inestabilidad política en América Latina. México: Fondo de Cultura Económica.

PNUD. (2004). La democracia en América Latina. Hacia una democracia de ciudadanas y ciudadanos. Informe y Contribuciones para un debate. Buenas Aires: Naciones Unidas.

PNUD. (2007). Hacia un Estado de y para la Democracia en América Latina. Buenos Aires: Naciones Unidas.

Poulos, J. (2012). Redistributive Preferences and Political Participation: Evidence from Norway. MIT: MIT Political Science Department Research Paper No. 2012-21.

Roberts, A., \& Garton Ash, T. (2009). Civil resistance and power politics: the experience of non-violent action from Gandhi to the present. New York: Oxford University Press.

RoberTs, K. M. (2017). Chilean Social Movements and Party Politics in Comparative Perspective: Conceptualizing Latin America's "Third Generation" of AntiNeoliberal Protest. In S. Donoso, \& M. Von Bülow, Social Movements in Chile. Organization, Trajectories, and Political Consequences (S. 221-247). New York: Palgrave-McMillan.

Rosanvallon, P. (2008). La contrademocracia: la politica en la era de la desconfianza. Madrid: Manantial.

SAder, E. (2008). El Estado. Posneoliberalismo en America Latina. Buenos Aires: CLACSO.

Schatzman, C. (2005). Political Challenge in Latin America: Rebellion and Collective Protest in an Era of Democratization . Journal of Peace Research (42)(3), 291-310.

Scholzman, K., Verba, S., \& Brady, H. (1999). Civic Participation in Equality Problem. In T. Skocpol, \& M. Fiorina, Civic Engagement in American Democracy (S. 427-459). Washington D.C: Brookings Institution Press.

Somma, N., \& Medel, R. (2017). Shifting relationship between social movements and institutional politics. In S. Donoso, \& M. Von Bülow, Social movements in Chile. Organization, trayectories and political consequences (S. 29-61). New York: Palgrave - McMillan.

Stiglitz, J. (2012). El precio de la dedigualdad. Madrid: Taurus.

Treviño, E., Béjares, C., Villalobos, C., \& Naranjo, E. (2017). Influence of teachers and schools on students civico outcomes in Latin America. Journal of Educational Research (110)(6), 604-618.

Valenzuela, S., Somma, N., Sherman, A., \& Arriagada, A. (2016). Social media in 
Latin America: deepening or brinding gaps in protest participation? Online Information Review (40) (5), 695-711.

Van Stekelenburg, J., \& Klandermans, B. (2013). The social psychology of protest. Current Sociology (61) (5-6), 886-905.

Veenstra, K., \& Haslam, A. (2000). Willingness to participate in industrial protest: Exploring social identification in context. British Journal of Social Psychology (39), 153-172.

Von Büllow, M. (2009). Networks of Trade Protest in the Americas: Toward a new labor internationalism? Latin American Politics and Society (51)(2), 1-28.

Wulff, S., Berstein, M., \& Taylor, V. (2015). New Theoretical Directions from the Study of Gender and Sexuality Movements: Collective Identity, MultiInstitutional Politics, and Emotions. In D. Della Porta, \& M. Diani, The Oxford Handbook of Social Movements. New York: Oxford University Press.

\section{Anexos}

\begin{tabular}{|c|c|c|}
\hline \multicolumn{3}{|c|}{ Anexo 1: Variables independientes consideradas } \\
\hline Variables & Operacionalización & $\begin{array}{l}\text { Efecto esperado en la } \\
\text { disposición de protestar }\end{array}$ \\
\hline Uso de Twitter & $\begin{array}{l}\text { Recodificación en dummy (1) Menciona } \\
\text { y (0) no menciona. Se excluye alternativa } \\
\text { NS/NR }\end{array}$ & + \\
\hline Uso Internet & $\begin{array}{l}\text { Variable categórica original, con la exclu- } \\
\text { sión de las alternativas NS/NR }\end{array}$ & + \\
\hline $\begin{array}{l}\text { Democracia es el mejor siste- } \\
\text { ma de gobierno }\end{array}$ & $\begin{array}{l}\text { Variable categórica, con exclusión de NS/ } \\
\text { NR }\end{array}$ & - \\
\hline $\begin{array}{l}\text { Grado de Democracia en el } \\
\text { país }\end{array}$ & $\begin{array}{l}\text { Variable escala, donde } 1 \text { ("No es demo- } \\
\text { crático") y } 10 \text { (Totalmente democrático). } \\
\text { Se excluyen NS/NR }\end{array}$ & - \\
\hline $\begin{array}{l}\text { Justicia en la Distribución del } \\
\text { Ingreso }\end{array}$ & $\begin{array}{l}\text { Variable categórica, recodificada en } \\
\text { "Muy justa" + "Justa" = } 1 \text {, "Injusta" + } \\
\text { "Muy injusta" = }\end{array}$ & - \\
\hline Recuerdo de Voto & $\begin{array}{l}\text { Recodificación en dummy, donde } 1= \\
\text { Voto en la última elección y } 0=\text { no votó. } \\
\text { Se excluyen respuestas NS/NR, no estaba } \\
\text { registrado o no recuerda. }\end{array}$ & + \\
\hline Escala izquierda-derecha & $\begin{array}{l}\text { Variable escala, donde } 0 \text { es "Izquierda" y } \\
10 \text { "Derecha". Se excluyen NS/NR }\end{array}$ & \\
\hline
\end{tabular}




\begin{tabular}{ll}
\hline Género & $\begin{array}{l}\text { Uso sin operacionalización de la pregunta }+ \\
\text { original }\end{array}$ \\
\hline Edad & $\begin{array}{l}\text { Uso sin operacionalización de la pregunta } \\
\text { original. Indica edad en años del entre- } \\
\text { vistado }\end{array}$ \\
\hline
\end{tabular}

\begin{tabular}{lll}
\hline Anexo 2: Probabilidades predichas (\%) & M5 & M6 \\
\hline Uso de Internet: Mucho & 46 & 46 \\
\hline Uso de Internet: Poco & 42 & 43 \\
\hline Uso de Internet: No usa & 33 & 34 \\
\hline Democracia: es el mejor sistema de gobierno & 42 & 42 \\
\hline $\begin{array}{l}\text { Democracia: no es el mejor sistema } \\
\text { (Referencia es el mejor) }\end{array}$ & 33 & 33 \\
\hline Democracia NS/NR (Referencia es el mejor) & 34 & 35 \\
\hline Votó en la última elección & 41 & 41 \\
\hline No votó en la última elección & 35 & 35 \\
\hline Hombres & 42 & 43 \\
\hline Mujeres & 38 & 38 \\
\hline
\end{tabular}

